\title{
Machine learning-based clinical outcome prediction in surgery for acromegaly
}

\author{
Olivier Zanier ${ }^{1} \cdot$ Matteo Zoli $^{2,3}$ - Victor E. Staartjes ${ }^{1}$ - Federica Guaraldi ${ }^{2}$ Sofia Asioli ${ }^{3,4}$ - Arianna Rustici ${ }^{5}$. \\ Valentino Marino Picciola ${ }^{6}$. Ernesto Pasquini ${ }^{7} \cdot$ Marco Faustini-Fustini ${ }^{2} \cdot$ Zoran Erlic $^{8} \cdot$ Luca Regli $^{1}$. \\ Diego Mazzatenta ${ }^{2,3} \cdot$ Carlo Serra ${ }^{1}{ }^{1}$
}

Received: 23 May 2021 / Accepted: 8 September 2021 / Published online: 12 October 2021

(c) The Author(s) 2021

\begin{abstract}
Purpose Biochemical remission (BR), gross total resection (GTR), and intraoperative cerebrospinal fluid (CSF) leaks are important metrics in transsphenoidal surgery for acromegaly, and prediction of their likelihood using machine learning would be clinically advantageous. We aim to develop and externally validate clinical prediction models for outcomes after transsphenoidal surgery for acromegaly.

Methods Using data from two registries, we develop and externally validate machine learning models for GTR, BR, and CSF leaks after endoscopic transsphenoidal surgery in acromegalic patients. For the model development a registry from Bologna, Italy was used. External validation was then performed using data from Zurich, Switzerland. Gender, age, prior surgery, as well as Hardy and Knosp classification were used as input features. Discrimination and calibration metrics were assessed.

Results The derivation cohort consisted of 307 patients (43.3\% male; mean [SD] age, 47.2 [12.7] years). GTR was achieved in $226(73.6 \%)$ and BR in $245(79.8 \%)$ patients. In the external validation cohort with 46 patients, 31 (75.6\%) achieved GTR and 31 (77.5\%) achieved BR. Area under the curve (AUC) at external validation was 0.75 (95\% confidence interval: 0.59-0.88) for GTR, $0.63(0.40-0.82)$ for BR, as well as $0.77(0.62-0.91)$ for intraoperative CSF leaks. While prior surgery was the most important variable for prediction of GTR, age, and Hardy grading contributed most to the predictions of BR and CSF leaks, respectively. Conclusions Gross total resection, biochemical remission, and CSF leaks remain hard to predict, but machine learning offers potential in helping to tailor surgical therapy. We demonstrate the feasibility of developing and externally validating clinical prediction models for these outcomes after surgery for acromegaly and lay the groundwork for development of a multicenter model with more robust generalization.
\end{abstract}

Keywords Pituitary $\cdot$ Predictive analytics $\cdot$ Outcome prediction $\cdot$ Machine learning $\cdot$ Acromegaly $\cdot$ Neurosurgery

These authors contributed equally: Olivier Zanier, Matteo Zoli

These authors jointly supervised this work: D. Mazzatenta, C. Serra

$\triangle$ Carlo Serra

c.serra@hotmail.it

1 Machine Intelligence in Clinical Neuroscience (MICN) Laboratory, Department of Neurosurgery, Clinical Neuroscience Center, University Hospital Zurich, University of Zurich, Zurich, Switzerland

2 IRCCS Istituto delle Scienze Neurologiche di Bologna, Programma Neurochirurgia Ipofisi-Pituitary Unit, Bologna, Italy

3 Department of Biomedical and Neuromotor Sciences (DIBINEM), University of Bologna, Bologna, Italy

4 Azienda USL di Bologna, Anatomic Pathology Unit,

\section{Introduction}

Acromegaly is a rare, progressive disease, caused by an oversecretion of growth hormone $(\mathrm{GH})$ and elevated levels of

Bologna, Italy

5 Department of Experimental, Diagnostic and Specialty Medicine (DIMES), University of Bologna, Bologna, Italy

6 University of Bologna, School of Medicine and Surgery, Bologna, Italy

7 Azienda USL di Bologna, Bellaria Hospital, ENT Unit, Bologna, Italy

8 Department of Endocrinology, Diabetology and Clinical Nutrition, University Hospital Zurich (USZ) and University of Zurich (UZH), Zurich, Switzerland 
insulin-like growth factor 1 (IGF-1) in the bloodstream [1]. A GH-secreting pituitary tumor is the cause of acromegaly in more than $95 \%$ of patients and surgical treatment remains the first-line therapy in most cases [2].

There are many variables that play into the likelihood of surgical success and endocrinological remission, such as age, Knosp grade, repeat surgeries, or even different somatostatin receptor subtypes [3-5]. The more factors that come into play, the harder it gets for clinicians to take them and their interactions into account. Based on these patient features, machine learning (ML) can be implemented to tailor treatment to a patient's individual characteristics in the era of "personalized medicine" [6]. It has become evident that ML has strong potential for outcome prediction and sometimes even outperforms statistical modeling techniques [7, 8].

The ability to predict the likelihood of outcomes such as gross total resection (GTR) and biochemical remission (BR) as well as complications that are clinically relevant such as intraoperative cerebrospinal fluid (CSF) leaks from simple information available pre-operatively would be beneficial in risk-benefit patient counseling and shared decision-making. For some complications such as intraoperative CSF leaks, modifiable risk factors could even be adjusted based on personal risk, and precautions such as lumbar drainage could be taken in individuals with a high predicted risk of CSF leaks [9]. For this reason, we aim to develop and externally validate clinical prediction models for outcomes after transsphenoidal surgery for acromegaly.

\section{Methods}

\section{Overview}

A registry of patients who underwent endoscopic transsphenoidal surgery from the Department of Neurosurgery, IRCCS Institute of Neurological Sciences of Bologna was used to train prediction models for GTR, BR, and intraoperative CSF leaks. External validation was then carried out using patient data from the Department of Neurosurgery, University Hospital Zurich. This study was conducted conforming to the methods of transparent reporting of a multivariable prediction model for individual prognosis or diagnosis (TRIPOD) statement [10].

\section{Data sources}

Prospective databases from two centers were used for model development. All patients who underwent surgery for acromegaly using the endoscopic transsphenoidal approach in Bologna from August 1998 to January 2020, as well as from July 2013 to May 2020 in Zurich were retrospectively evaluated. Operative procedures and preoperative assessments were conducted as described in previous publications [11, 12]. The inclusion criterion was for one or more of the three outcome measures (GTR, BR, and CSF leaks) to be available. Exclusion criteria were transcranial or combined procedures.

\section{Outcome measures}

The ML models were developed to predict the subsequent binary endpoints: GTR, BR, and intraoperative CSF leaks. The primary outcome was GTR. The extent of resection was measured in a 3-month postoperative volumetric MRI and calculated as the percentage-wise reduction of tumor volume compared to baseline tumor volume on preoperative MRI. An extent of resection of $100 \%$ was defined as GTR. All measurements were performed by a board-certified neurosurgeon with extensive experience in pituitary surgery and imaging and were continually entered into the prospective registry. BR was strictly defined as normalization of hypersecretion into the normal reference range as defined by accepted international guidelines [13]. BR was defined as postoperative $\mathrm{HGH}$ level random or after oral glucose tolerance test $<0.4 \mu \mathrm{g} / \mathrm{l}$ with normalization of age-adjusted IGF-1 levels at least 12 weeks after surgery and no clinical signs of $\mathrm{GH}$ activity. Cases with persistent slightly elevation of IGF-1 levels were considered in remission if $\mathrm{HGH}$ level after OGTT was adequately suppressed and no clinical signs of hormonal activity were present. The HGH and IGF-1 were measured using the chemiluminescence-Immunoassay LIAISON $^{\infty}$ hGH and LIAISON $^{\oplus}$ IGF-I, respectively. The analyses were performed on the Liaison XL-Machine (DiaSorin, Saluggia, Italy).

Note that supplemental treatment modalities such as medical and radiation therapy were also taken into account when calculating BR.

\section{Input variables}

Furthermore, we collected the following baseline variables: age, gender, prior surgery, Hardy classification (sellar and suprasellar) [14], Knosp classification [15], and tumor size. The Hardy and Knosp classifications both describe tumor morphology and correlate with resectability: While the Hardy classification focuses on intrasellar growth patterns and suprasellar extension, the Knosp classification assesses risk of cavernous sinus invasion by considering parasellar tumor extension relative to the internal carotid arteries $[14,15]$. We defined macroadenoma as tumor size greater than $10 \mathrm{~mm}$ [16].

\section{Model development and validation}

Continuous data are reported as mean value \pm standard deviation (SD), while categorical data are given as absolute numbers (percentages). Models were trained on data from Bologna, and subsequently externally validated in Zurich. 
Table 1 Patient characteristics and incidence of outcomes

\begin{tabular}{|c|c|c|}
\hline \multirow[t]{2}{*}{ Variable } & \multicolumn{2}{|l|}{ Cohort } \\
\hline & $\begin{array}{l}\text { Development } \\
(n=307)\end{array}$ & $\begin{array}{l}\text { External validation } \\
(n=46)\end{array}$ \\
\hline Male gender, $n(\%)$ & $133(43.3 \%)$ & $22(47.8 \%)$ \\
\hline No. missing & $0(0.0 \%)$ & $0(0.0 \%)$ \\
\hline \multicolumn{3}{|l|}{ Age [yrs.] } \\
\hline Mean \pm SD & $47.2 \pm 12.7$ & $47.5 \pm 14.4$ \\
\hline Median (IQR) & $55(38-57)$ & $46(37-60)$ \\
\hline Range & $13-78$ & $21-73$ \\
\hline No. missing & $1(0.3 \%)$ & $0(0.0 \%)$ \\
\hline Prior surgery, $n(\%)$ & $49(16 \%)$ & $10(21.7 \%)$ \\
\hline No. missing & $0(0.0 \%)$ & $0(0.0 \%)$ \\
\hline Hardy sellar, $n(\%)$ & $236(76.9 \%)$ & $42(91.3 \%)$ \\
\hline Grade 1 & $514(21.1 \%)$ & $14(30.4 \%)$ \\
\hline Grade 2 & $324(13.3 \%)$ & $10(21.7 \%)$ \\
\hline Grade 3 & $243(10.0 \%)$ & $3(6.5 \%)$ \\
\hline Grade 4 & $121(5.0 \%)$ & $15(32.6 \%)$ \\
\hline No. missing & $0(0.0 \%)$ & $3(6.5 \%)$ \\
\hline Hardy suprasellar, $n(\%)$ & $174(56.7 \%)$ & $21(45.6 \%)$ \\
\hline Grade A & $109(35.5 \%)$ & $13(28.3 \%)$ \\
\hline Grade B & $20(6.5 \%)$ & $6(13.0 \%)$ \\
\hline Grade C & $2(0.7 \%)$ & $1(2.2 \%)$ \\
\hline Grade D & $3(1 \%)$ & $0(0 \%)$ \\
\hline Grade E & $40(13 \%)$ & $1(2.2 \%)$ \\
\hline No. missing & $0(0.0 \%)$ & $1(2.2 \%)$ \\
\hline Knosp classification, $n(\%)$ & $96(31.3 \%)$ & $31(67.4 \%)$ \\
\hline Grade 1 & $24(7.8 \%)$ & $7(15.2 \%)$ \\
\hline Grade 2 & $27(8.8 \%)$ & $6(13.0 \%)$ \\
\hline Grade 3 & $30(9.8 \%)$ & $15(32.6 \%)$ \\
\hline Grade 4 & $15(4.9 \%)$ & $3(6.5 \%)$ \\
\hline No. missing & $0(0.0 \%)$ & $0(0.0 \%)$ \\
\hline Macroadenoma, $n(\%)$ & $199(64.8 \%)$ & $36(80.0 \%)$ \\
\hline No. missing & $0(0.0 \%)$ & $1(2.2 \%)$ \\
\hline Gross total resection (GTR), $n(\%)$ & $226(73.6 \%)$ & $31(75.6 \%)$ \\
\hline No. missing & $0(0.0 \%)$ & $5(10.9 \%)$ \\
\hline Intraop. CSF leak, $n(\%)$ & $38(12.5 \%)$ & $12(26.1 \%)$ \\
\hline No. missing & $0(0.0 \%)$ & $0(0.0 \%)$ \\
\hline Biochemical remission, $n(\%)$ & $245(79.8 \%)$ & $31(77.5 \%)$ \\
\hline No. missing & $0(0.0 \%)$ & $6(13.0 \%)$ \\
\hline
\end{tabular}

$S D$ standard deviation, $I Q R$ interquartile range

Both data sets were randomly reordered and then checked for approximately equal class distribution. There was no need for recursive feature elimination as only a limited number of variables were purposefully used.

A wide range of ML algorithms was applied including traditional and Bayesian generalized linear models (GLM), generalized additive models, random forests, stochastic gradient boosting machines (GBM) and a shallow neural network. They were tuned according to the area under the receiver operating characteristics curve (AUC) in fivefold repeated cross validation with ten iterations. A k-nearest neighbor algorithm was trained in parallel, allowing imputation of any missing data [17]. Binarization of predicted probabilities was carried out using a threshold based on the closest-to-(0,1)-criterion [18] on the derivation cohort. Discrimination was assessed using AUC, accuracy, sensitivity, specificity, positive predictive value (PPV), and negative predictive value (NPV). We also assessed calibration intercept and slope. Nonparametric $95 \%$ confidence intervals (CI) of the discrimination and calibration metrics were computed in 1000 bootstrap resamples. Variable importance was assessed for each model using a universal AUC-based method, and importance measures were scaled from 0 to 100 for each model [19]. All evaluations were executed using $\mathrm{R}$ version 4.0.2 [20].

\section{Results \\ Patient cohort}

In total, 307 patients were used in the training process. The training data had no missing values apart from the age of a single patient. Mean age was $47.2 \pm 12.7$ years and 133 (43.3\%) patients were male. GTR and BR were achieved in $226(73.6 \%)$ and $245(79.8 \%)$ patients, respectively, and CSF leaks occurred intraoperative in $38(12.5 \%)$ of patients. The external validation cohort consisted of 46 patients, among whom there were $5(10.9 \%)$ patients with incomplete data on GTR and $6(13.0 \%)$ patients with incomplete data on BR. Only four $(8.7 \%)$ patients in the external validation set had incomplete baseline data. Mean age was $47.5 \pm 14.4$ years and $22(47.8 \%)$ patients were male. In the external validation cohort, GTR occurred in $31(75.6 \%)$ patients, while BR occurred in $31(77.5 \%)$. Intraoperative CSF leaks occurred in $12(26.1 \%)$ patients in the external validation cohort. Detailed patient characteristics for both cohorts are provided in Table 1.

\section{Model performance}

\section{Gross total resection}

A detailed overview of model performance is provided in Table 2, including calibration metrics and training performance. At external validation, the GTR model (traditional GLM) achieved an AUC of 0.75 (0.59-0.88), 0.52 (0.33-0.70) for sensitivity and $0.90(0.69-1.00)$ for specificity. The resulting PPV was 0.94 (0.82-1.00).

\section{Biochemical remission}

Our GBM achieved an AUC of $0.63(0.40-0.82)$ on the external validation data, as well as a sensitivity of 0.61 $(0.44-0.77)$ and specificity of $0.44(0.12-0.80)$. A PPV of $0.79(0.61-0.95)$ was reached. 
Table 2 Discrimination and calibration metrics of the machine learning-based prediction models

\begin{tabular}{|c|c|c|c|c|c|c|}
\hline \multirow{3}{*}{$\begin{array}{l}\text { Outcome } \\
\text { Type of model } \\
\text { Metric }\end{array}$} & \multicolumn{2}{|c|}{ Gross total resection } & \multicolumn{2}{|c|}{ Biochemical remission } & \multicolumn{2}{|l|}{ CSF leak } \\
\hline & \multicolumn{2}{|l|}{ GLM } & \multicolumn{2}{|l|}{ GBM } & \multicolumn{2}{|l|}{ Bayesian GLM } \\
\hline & $\begin{array}{l}\text { Development } \\
(n=307)\end{array}$ & $\begin{array}{l}\text { External validation } \\
(n=41)\end{array}$ & $\begin{array}{l}\text { Development } \\
(n=307)\end{array}$ & $\begin{array}{l}\text { External validation } \\
(n=46)\end{array}$ & $\begin{array}{l}\text { Development } \\
(n=307)\end{array}$ & $\begin{array}{l}\text { External validation } \\
(n=40)\end{array}$ \\
\hline \multicolumn{7}{|l|}{ Discrimination } \\
\hline AUC & $0.68(0.66-0.70)$ & $0.75(0.59-0.88)$ & $0.62(0.59-0.64)$ & $0.63(0.40-0.82)$ & $0.69(0.67-0.72)$ & $0.77(0.62-0.91)$ \\
\hline Accuracy & $0.65(0.63-0.67)$ & $0.61(0.46-0.75)$ & $0.63(0.61-0.64)$ & $0.58(0.42-0.72)$ & $0.60(0.58-0.62)$ & $0.70(0.57-0.83)$ \\
\hline Sensitivity & $0.65(0.63-0.67)$ & $0.52(0.33-0.70)$ & $0.64(0.63-0.67)$ & $0.61(0.44-0.77)$ & $0.71(0.66-0.75)$ & $0.58(0.29-0.87)$ \\
\hline Specificity & $0.65(0.61-0.68)$ & $0.90(0.69-1.00)$ & $0.57(0.53-0.61)$ & $0.44(0.12-0.80)$ & $0.59(0.57-0.61)$ & $0.74(0.57-0.88)$ \\
\hline PPV & $0.84(0.82-0.85)$ & $0.94(0.82-1.00)$ & $0.85(0.84-0.87)$ & $0.79(0.61-0.95)$ & $0.19(0.17-0.22)$ & $0.44(0.20-0.69)$ \\
\hline NPV & $0.40(0.37-0.43)$ & $0.38(0.18-0.57)$ & $0.27(0.26-0.31)$ & $0.25(0.06-0.47)$ & $0.93(0.92-0.95)$ & $0.83(0.69-0.96)$ \\
\hline \multicolumn{7}{|l|}{ Calibration } \\
\hline Intercept & 0.97 & 1.49 & 1.29 & 1.14 & -1.77 & -0.64 \\
\hline Slope & 0.52 & 0.03 & 0.58 & 0.76 & 0.39 & 0.68 \\
\hline Threshold & 0.55 & & 0.52 & & 0.41 & \\
\hline
\end{tabular}

Metrics are provided with bootstrapped $95 \%$ confidence intervals

$A U C$ area under the curve, $P P V$ positive predictive value, $N P V$ negative predictive value

Table 3 AUC-based relative variable importance in the machine learning-based prediction models

\begin{tabular}{lccc}
\hline Variable & $\begin{array}{l}\text { Gross total } \\
\text { resection }\end{array}$ & $\begin{array}{l}\text { Biochemical } \\
\text { remission }\end{array}$ & CSF leaks \\
\hline Male gender & 58.24 & $0.00^{*}$ & $0.00^{*}$ \\
Age & 56.51 & 100.00 & 12.11 \\
Prior surgery & 100.00 & 2.71 & 14.37 \\
Hardy sellar & & & 47.47 \\
Grade 1 & 0.02 & 9.36 & \\
Grade 2 & 25.16 & 1.93 & \\
Grade 3 & 51.03 & $0.00^{*}$ & \\
Grade 4 & 0.30 & $0.00^{*}$ & \\
Hardy suprasellar & & & \\
Grade A & 90.49 & 2.67 & \\
Grade B & 93.01 & 13.21 & \\
Grade C & $0.00^{*}$ & $0.00^{*}$ & \\
Grade D & 0.12 & $0.00^{*}$ & \\
Grade E & 72.18 & 14.77 & 67.41 \\
Knosp classification & & & \\
Grade 1 & 6.71 & $0.00^{*}$ & \\
Grade 2 & 63.30 & 4.81 & \\
Grade 3 & 25.54 & 0.00 & \\
Grade 4 & 8.19 & 15.14 & \\
Macroadenoma & 21.24 & 7.48 & \\
\hline
\end{tabular}

*Corresponds to a variable importance of 0.00 , i.e., the variable was not included in the final model

\section{Cerebrospinal fluid leaks}

The Bayesian GLM used to predict CSF leaks displayed an AUC of $0.77(0.62-0.91)$ at external validation, while a sensitivity of $0.58(0.29-0.87)$ and a specificity of 0.74 $(0.57-0.88)$ were recorded. The NPV reached 0.83 (0.69-0.96).

\section{Variable importance}

Table 3 provides an overview of variable importance measures for each of the three models. For prediction of GTR, prior surgery and Hardy grading contributed most to predictions. Patient age and Hardy grading contributed most to predictions of BR. Last, Hardy and Knosp grading contributed most to predictions of intraoperative CSF leaks (Fig. 1).

\section{Discussion}

In this study, the feasibility of predicting surgical and endocrinological outcome after transsphenoidal surgical treatment of acromegaly was evaluated. With data from two registries, three clinical prediction models were trained and subsequently externally validated. The achieved results proved to be promising and thereby displayed that there is significant potential for clinical application of ML.

In surgical treatment of acromegaly, normalization of GH levels through total resection is crucial. Treatmentrefractory acromegaly puts patients at risk for early mortality [21]. Consequently, a more aggressive surgical approach is justified in refractory cases. It has been proven that the percentage of reduction in GH closely correlates with the fraction of removed tumor in surgery for acromegaly [22]. Further, low serum GH levels indicate persisting remission, whereas with higher levels the probability of recurrent disease-linked to significant mortality -is markedly larger [23, 24]. Even intraoperative CSF leaks are detrimental to endocrinological outcomes, since they have 

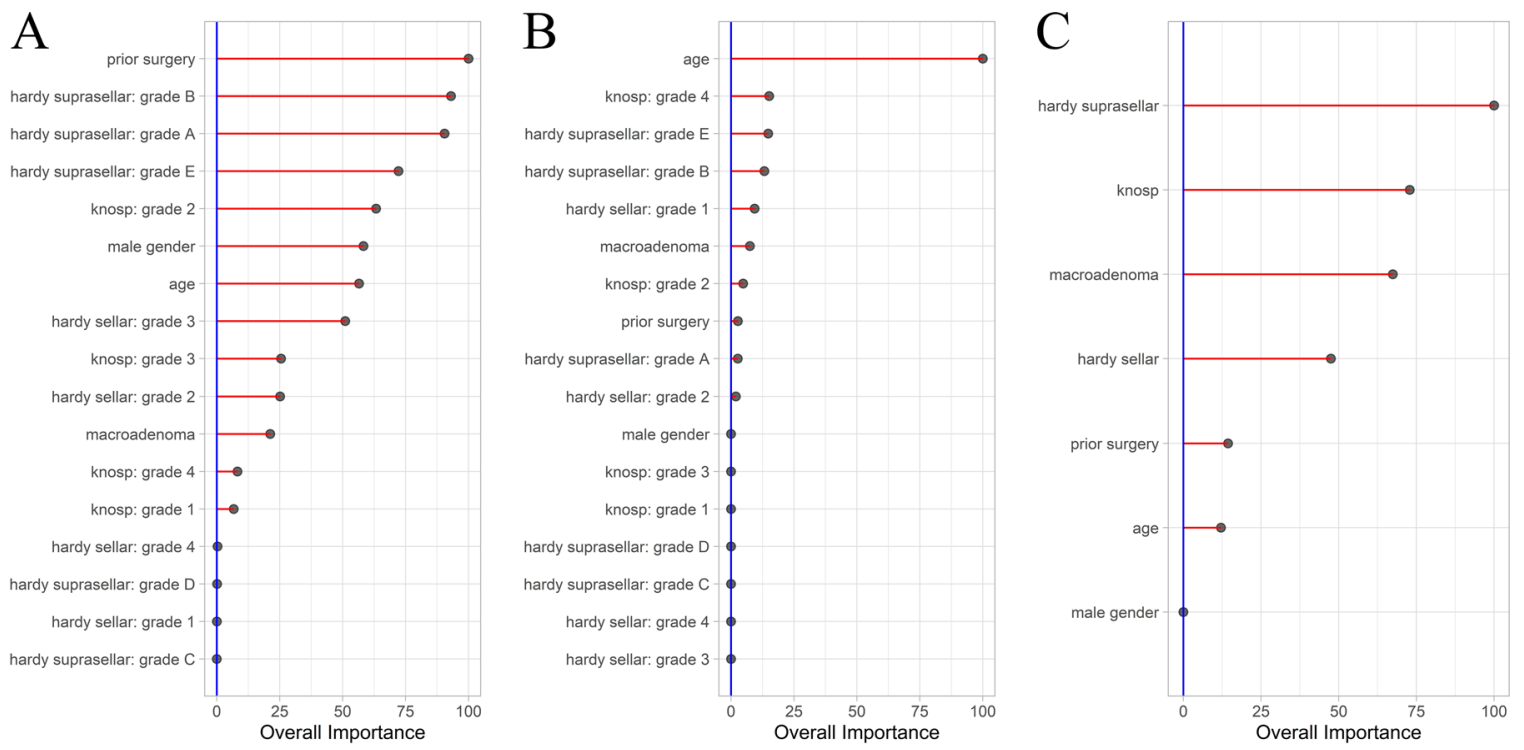

Fig. 1 AUC-based variable importance for the three models. Importance values have been scaled from 0 to 100 . A Gross total resection; B Biochemical remission; C Intraoperative cerebrospinal fluid leakage

been shown to inhibit hormonal recovery after surgeryapart from their inherent risk for persisting CSF fistulas and meningitis $[25,26]$.

Surgical outcome depends on many variables that are hard to account for-including surgical experience, skill, and caseload [2] - making their prediction difficult. ML methods can deduce a simple risk assessment model from relatively complex data $[4,5]$. For this reason, ML has been proven to aid in improved shared decision-making as well as enhanced patient care by modification of risk factors [7, 27, 28]. However, some factors cannot be taken into account by any model-prediction models will always remain just that: models of reality. Therefore, ML should never replace the careful study of imaging results, the contemporary literature, and surgical experience. Rather, it should be seen as supplemental information available to surgeons, complementing the existing evidence and allowing personalized risk-benefit assessment. There is decent evidence that ML can help with improved surgical decisionmaking, and in some cases may even outperform expert predictions [28].

Other important parameters that help physicians include simple scores and classifications, like the Knosp classification [29] or the Zurich Pituitary Score [30]. While these scores are well validated and robustly predict e.g. GTR, they are rather difficult to tailor to specific patient characteristics because they stratify patients into large risk groups. For the ML models established in this investigation, some of these classifications were combined with other recognized prognostic factors to deliver predictions that are precisely tailored to each patient. When trying to compare the performance of ML models with these scoring systems, little valid comparisons can be made, since reporting of performance measures such as sensitivity and specificity for these scores is uncommon. A systematic review by Dhandapani et al. [31] allows comparison to the raw Knosp classification and its relationship with GTR. This review found that the usual dichotomization of the Knosp classification (Knosp 1 and 2 vs. Knosp 3 and 4) led to a sensitivity of $66.4 \%$ and specificity of $90.3 \%$ for GTR [31]. Furthermore, in future studies, by combining additional endocrinological parameters like preoperative IGF-1 or early postoperative GH value in the model, a better performance for BR prediction might be obtained [32-34]. However, the rationale of this study was to develop a simple tool that can give meaningful predictions using basic, preoperatively available data only.

The developed models demonstrated good generalizability, performing similarly well on the external validation data as compared to on the training data. The GTR and BR models had a high PPV, making them suitable as "rulein" models. Conversely, the CSF leak model demonstrated a high NPV, and is thus more suitable in a "rule-out" setting.

A major criticism of ML-based prediction models is that they at times work like a "black box" [35]. Especially with deep neural networks, one is often confronted with the inability to understand why certain predictions have been made. By feeding the algorithm with the required data it can often provide precise outcome prediction, but it remains unknown how the internal decision-making process works. In this study, an initial problem was solved firstly by relying on algorithms with a complexity suitable to tabulated medical data. In addition, insight into the decision-making procedure can be gained by evaluating the variable 
importance listed in Table 3. With ML, interpretability can involve an inherent trade-off for better prediction power.

In conclusion, it can be stated that prediction of these complex outcomes like BR and GTR-which are certainly governed also by "unmeasurable" factors such as surgeon experience-from simple input data remains a difficult task, although ML can provide relatively accurate predictions in this pilot study already. Using more complex variables as input instead would probably improve the performance, but too complex inputs could be undesirable, as they would make the application of the models impractical. This study aimed at creating a simple tool that can give meaningful predictions using basic, pre-operatively available data. The models developed are proof that this is no longer mere wishful thinking. To the best of the authors' knowledge, there are no other published, externally validated clinical prediction models for outcomes of transsphenoidal pituitary surgery in acromegalic patients. Once these models are enhanced by additional patient data and more participating centers to foster generalizability, an integration into a web application available to the public would be feasible.

\section{Limitations}

The main limitation of our study is the relatively low sample size. Although a very decent surgical cohort of over 300 acromegalic patients was included for training - one of the largest contemporary single-center cohorts in the literaturethis sample size is still rather low for ML. For example, evaluation of model calibration usually requires larger amounts of data. Recalibration would not change anything in this respect, and would only artificially improve calibration $[36,37]$. Larger amounts of data would also likely improve general model performance. Even though external validation was carried out, which demonstrated generalizability of our models, including more participating centers to create a multicenter model that may account for the differences in surgical strategies, and so forth. Another important factor to consider is that these models are not applicable to centers with radically different treatment protocols. Importantly, surgical outcomes are also influenced by surgical experience and caseload [38], inherently limiting the generalizability of any prediction model, score, or classification for surgical outcome. For example, a significantly different endpoint incidence may lead to systematic over- or underestimation of the outcome probability from the developed models [36]. Furthermore, it needs to be taken into account that all clinical prediction models are unable to reliably predict extreme cases that fall outside the range of the training data (extrapolation) $[39,40]$. Furthermore, our models are trained on "real-world" registry data. The rate of BR was higher than the rate of GTR due to supplemental treatments such as radiation and medical therapy. While this does represent the "real-world" clinical practice-with some patients undergoing multiple treatments - our models may be less suitable when aiming to predict the chances of BR purely from tumor resection. Problems may also occur because of the poor reliability between different physicians' ratings [41, 42]. Especially with the Knosp and Hardy classification, there is evidence for poor inter-rater reliability.

\section{Conclusions}

GTR, BR, and CSF leaks remain hard to predict, but ML may offer remarkable potential in helping to tailor surgical therapy. We demonstrate the feasibility of developing and externally validating clinical prediction models for these outcomes after surgery for acromegaly. This study lays the groundwork for development of a multicenter model with more robust generalization.

\section{Data availability}

The data in support of our findings can be obtained upon reasonable request from the corresponding author.

\section{Code availability}

For the source code used to compute the presented results refer to the Supplement.

Funding Open Access funding provided by Universität Zürich.

\section{Compliance with ethical standards}

Conflict of interest The authors declare no competing interests.

Ethical approval Patient data were treated according to the ethical standards of the Declaration of Helsinki and its amendments as approved by our institutional committee (Cantonal Ethics Committee Zürich, KEK St-V-Nr 2015-0242) and the interhospital Ethical Committee of Bologna City (protocol CE17143, February 2018).

Publisher's note Springer Nature remains neutral with regard to jurisdictional claims in published maps and institutional affiliations.

Open Access This article is licensed under a Creative Commons Attribution 4.0 International License, which permits use, sharing, adaptation, distribution and reproduction in any medium or format, as long as you give appropriate credit to the original author(s) and the source, provide a link to the Creative Commons license, and indicate if changes were made. The images or other third party material in this article are included in the article's Creative Commons license, unless indicated otherwise in a credit line to the material. If material is not included in the article's Creative Commons license and your intended use is not permitted by statutory regulation or exceeds the permitted use, you will need to obtain permission directly from the copyright holder. To view a copy of this license, visit http://creativecommons. org/licenses/by/4.0/. 


\section{References}

1. R. Dineen, P.M. Stewart, M. Sherlock, Acromegaly. QJM Mon. J. Assoc. Physicians 110, 411-420 (2017). https://doi.org/10.1093/ qjmed/hcw004

2. M. Buchfelder, S.-M. Schlaffer, The surgical treatment of acromegaly. Pituitary 20, 76-83 (2017). https://doi.org/10.1007/ s11102-016-0765-7

3. H. Sun, J. Brzana, C.G. Yedinak, S.H. Gultekin, J.B. Delashaw, M. Fleseriu, Factors associated with biochemical remission after microscopic transsphenoidal surgery for acromegaly. J. Neurol. Surg. B Skull Base 75, 47-52 (2014). https://doi.org/10.1055/s0033-1354578

4. E.C. Coopmans, M.R. Postma, T.L.C. Wolters, S.W.F. van Meyel, R. Netea-Maier, A.P. van Beek, S.J.C.M.M. Neggers, Predictors for remission after transsphenoidal surgery in acromegaly: a Dutch Multicenter Study. J. Clin. Endocrinol. Metab. 106, 1783-1792 (2021). https://doi.org/10.1210/clinem/dgab069

5. M. Fleseriu, B.M.K. Biller, P.U. Freda, M.R. Gadelha, A. Giustina, L. Katznelson, M.E. Molitch, S.L. Samson, C.J. Strasburger, A.J. van der Lely, S. Melmed, A Pituitary Society update to acromegaly management guidelines. Pituitary 24, 1-13 (2021). https://doi.org/10.1007/s11102-020-01091-7

6. Z. Obermeyer, E.J. Emanuel, Predicting the future - big data, machine learning, and clinical medicine. N. Engl. J. Med. 375, 1216-1219 (2016). https://doi.org/10.1056/NEJMp1606181

7. J.T. Senders, P.C. Staples, A.V. Karhade, M.M. Zaki, W.B. Gormley, M.L.D. Broekman, T.R. Smith, O. Arnaout, Machine learning and neurosurgical outcome prediction: a systematic review. World Neurosurg. 109, 476-486.e1 (2018). https://doi. org/10.1016/j.wneu.2017.09.149

8. V.E. Staartjes, C. Serra, G. Muscas, N. Maldaner, K. Akeret, C.H. B. van Niftrik, J. Fierstra, D. Holzmann, L. Regli, Utility of deep neural networks in predicting gross-total resection after transsphenoidal surgery for pituitary adenoma: a pilot study. Neurosurg. Focus 45, E12 (2018). https://doi.org/10.3171/2018.8. FOCUS18243

9. G.U. Mehta, E.H. Oldfield, Prevention of intraoperative cerebrospinal fluid leaks by lumbar cerebrospinal fluid drainage during surgery for pituitary macroadenomas: clinical article. J. Neurosurg. 116, 1299-1303 (2012). https://doi.org/10.3171/2012.3.JNS112160

10. G.S. Collins, J.B. Reitsma, D.G. Altman, K.G.M. Moons, Transparent reporting of a multivariable prediction model for individual prognosis or diagnosis (TRIPOD): the TRIPOD statement. Ann. Intern. Med. 162, 55-63 (2015). https://doi.org/10. 7326/M14-0697

11. N. Maldaner, C. Serra, O. Tschopp, C. Schmid, O. Bozinov, L. Regli, Modernes Management von Hypophysenadenomen gegenwärtiger Stand in Diagnostik, Therapie und Nachsorge. Praxis 107, 825-835 (2018). https://doi.org/10.1024/1661-8157/a 003035

12. C. Serra, J.-K. Burkhardt, G. Esposito, O. Bozinov, A. Pangalu, A. Valavanis, D. Holzmann, C. Schmid, L. Regli, Pituitary surgery and volumetric assessment of extent of resection: a paradigm shift in the use of intraoperative magnetic resonance imaging. Neurosurg. Focus 40, E17 (2016). https://doi.org/10.3171/2015. 12.FOCUS15564

13. A. Giustina, G. Barkhoudarian, A. Beckers, A. Ben-Shlomo, N. Biermasz, B. Biller, C. Boguszewski, M. Bolanowski, J. Bollerslev, V. Bonert, M.D. Bronstein, M. Buchfelder, F. Casanueva, P. Chanson, D. Clemmons, M. Fleseriu, A.M. Formenti, P. Freda, M. Gadelha, E. Geer, M. Gurnell, A.P. Heaney, K.K.Y. Ho, A.G. Ioachimescu, S. Lamberts, E. Laws, M. Losa, P. Maffei, A. Mamelak, M. Mercado, M. Molitch, P. Mortini, A.M. Pereira, S. Petersenn, K. Post, M. Puig-Domingo, R. Salvatori, S.L. Samson,
I. Shimon, C. Strasburger, B. Swearingen, P. Trainer, M.L. Vance, J. Wass, M.E. Wierman, K.C.J. Yuen, M.C. Zatelli, S. Melmed, Multidisciplinary management of acromegaly: a consensus. Rev. Endocr. Metab. Disord. 21, 667-678 (2020). https://doi.org/10. 1007/s11154-020-09588-Z

14. J. Hardy, J.L. Vezina, Transsphenoidal neurosurgery of intracranial neoplasm. Adv. Neurol. 15, 261-273 (1976)

15. E. Knosp, E. Steiner, K. Kitz, C. Matula, Pituitary adenomas with invasion of the cavernous sinus spacea magnetic resonance imaging classification compared with surgical findings. Neurosurgery 33, 610-618 (1993). https://doi.org/10.1097/00006123-199310000-00008

16. J.A. Jane, M.P. Catalino, E.R. Laws, in Surgical Treatment of Pituitary Adenomas, ed. by K.R. Feingold, B. Anawalt, A. Boyce, G. Chrousos, W.W. de Herder, K. Dungan, A. Grossman, J.M. Hershman, H.J. Hofland, G. Kaltsas, C. Koch, P. Kopp, M. Korbonits, R. McLachlan, J.E. Morley, M. New, J. Purnell, F. Singer, C.A. Stratakis, D.L. Trence, D.P. Wilson. Endotext. (MDText.com, Inc.: South Dartmouth (MA), 2000)

17. G.E.A.P.A. Batista, M.C. Monard, An analysis of four missing data treatment methods for supervised learning. Appl. Artif. Intell. 17, 519-533 (2003). https://doi.org/10.1080/713827181

18. N.J. Perkins, E.F. Schisterman, The inconsistency of "optimal" cutpoints obtained using two criteria based on the receiver operating characteristic curve. Am. J. Epidemiol. 163, 670-675 (2006). https://doi.org/10.1093/aje/kwj063

19. Kuhn, M. Building predictive models in $R$ using the caret package. J. Stat. Softw. 28 (2008). https://doi.org/10.18637/jss.v028.i05

20. R Core Team. R: A Language and Environment for Statistical Computing. Vienna, Austria: R Foundation for Statistical Computing, 2021. https://www.R-project.org/

21. M.E. Sughrue, E.F. Chang, R.A. Gabriel, M.K. Aghi, L.S. Blevins, Excess mortality for patients with residual disease following resection of pituitary adenomas. Pituitary 14, 276-283 (2011). https://doi.org/10.1007/s11102-011-0308-1

22. L. Schwyzer, R.M. Starke, J.A. Jane, E.H. Oldfield, Percent reduction of growth hormone levels correlates closely with percent resected tumor volume in acromegaly. J. Neurosurg. 122, 798-802 (2015). https://doi.org/10.3171/2014.10.JNS14496

23. I.M. Holdaway, M.J. Bolland, G.D. Gamble, A meta-analysis of the effect of lowering serum levels of GH and IGF-I on mortality in acromegaly. Eur. J. Endocrinol. 159, 89-95 (2008). https://doi. org/10.1530/EJE-08-0267

24. M.D. Krieger, W.T. Couldwell, M.H. Weiss, Assessment of longterm remission of acromegaly following surgery. J. Neurosurg. 98, 719-724 (2003). https://doi.org/10.3171/jns.2003.98.4.0719

25. N. Fatemi, J.R. Dusick, C. Mattozo, D.L. McArthur, P. Cohan, J. Boscardin, C. Wang, R.S. Swerdloff, D.F. Kelly, Pituitary hormonal loss and recovery after transsphenoidal adenoma removal. Neurosurgery 63, 709-718 (2008). https://doi.org/10.1227/01. NEU.0000325725.77132.90. discussion 718-719

26. V.E. Staartjes, C.M. Zattra, K. Akeret, N. Maldaner, G. Muscas, C.H. Bas van Niftrik, J. Fierstra, L. Regli, C. Serra, Neural network-based identification of patients at high risk for intraoperative cerebrospinal fluid leaks in endoscopic pituitary surgery. J. Neurosurg. 1-7 (2019). https://doi.org/10.3171/2019.4. JNS19477

27. M. Broggi, C.M. Zattra, F. Vasella, J. Velz, S. Schiavolin, C. Serra, J. Bartek, A. Fletcher-Sandersjöö, P. Förander, D. Kalasauskas, M. Renovanz, F. Ringel, K.R. Brawanski, J. Kerschbaumer, C.F. Freyschlag, A.S. Jakola, K. Sjåvik, O. Solheim, B. Schatlo, A. Sachkova, H.C. Bock, A. Hussein, V. Rohde, M.L. D. Broekman, C.O. Nogarede, C.M.C. Lemmens, J.M. Kernbach, G. Neuloh, O. Bozinov, N. Krayenbühl, J. Sarnthein, P. Ferroli, L. Regli, M.N. Stienen, FEBNS, Development and external validation of a clinical prediction model for functional impairment after 
intracranial tumor surgery. J. Neurosurg. 1-8 (2020). https://doi. org/10.3171/2020.4.JNS20643

28. J.T. Senders, O. Arnaout, A.V. Karhade, H.H. Dasenbrock, W.B. Gormley, M.L. Broekman, T.R. Smith, Natural and artificial intelligence in neurosurgery: a systematic review. Neurosurgery 83, 181-192 (2018). https://doi.org/10.1093/neuros/nyx384

29. M. Buchy, V. Lapras, M. Rabilloud, A. Vasiljevic, F. BorsonChazot, E. Jouanneau, G. Raverot, Predicting early post-operative remission in pituitary adenomas: evaluation of the modified knosp classification. Pituitary 22, 467-475 (2019). https://doi.org/10. 1007/s11102-019-00976-6

30. V.E. Staartjes, C. Serra, M. Zoli, D. Mazzatenta, F. Pozzi, D. Locatelli, E. D'Avella, D. Solari, L.M. Cavallo, L. Regli, Multicenter external validation of the Zurich Pituitary Score. Acta Neurochir. 162, 1287-1295 (2020). https://doi.org/10.1007/ s00701-020-04286-w

31. S. Dhandapani, H. Singh, H.M. Negm, S. Cohen, V.K. Anand, T. H. Schwartz, Cavernous sinus invasion in pituitary adenomas: systematic review and pooled data meta-analysis of radiologic criteria and comparison of endoscopic and microscopic surgery. World Neurosurg. 96, 36-46 (2016). https://doi.org/10.1016/j. wneu.2016.08.088

32. P. Dutta, M. Korbonits, N. Sachdeva, P. Gupta, A. Srinivasan, J.S. Devgun, A. Bajaj, K.K. Mukherjee, Can immediate postoperative random growth hormone levels predict long-term cure in patients with acromegaly? Neurol. India 64, 252-258 (2016). https://doi. org/10.4103/0028-3886.177622

33. R. Rotermund, T. Burkhardt, Z. Rohani, R. Jung, J. Aberle, J. Flitsch, Value of early postoperative random growth hormone levels and nadir growth hormone levels after oral glucose tolerance testing in acromegaly. Growth Horm. IGF Res. 41, 64-70 (2018). https://doi.org/10.1016/j.ghir.2018.03.002

34. E.H. Kim, M.C. Oh, E.J. Lee, S.H. Kim, Predicting long-term remission by measuring immediate postoperative growth hormone levels and oral glucose tolerance test in acromegaly. Neurosurgery
70, 1106-1113 (2012). https://doi.org/10.1227/NEU.0b013e31823 f5c16. discussion 1113

35. C. Rudin, Stop explaining black box machine learning models for high stakes decisions and use interpretable models instead. Nat. Mach. Intell. 1, 206-215 (2019). https://doi.org/10.1038/s42256019-0048-x

36. K.J.M. Janssen, K.G.M. Moons, C.J. Kalkman, D.E. Grobbee, Y. Vergouwe, Updating methods improved the performance of a clinical prediction model in new patients. J. Clin. Epidemiol. 61, 76-86 (2008). https://doi.org/10.1016/j.jclinepi.2007.04. 018

37. V.E. Staartjes, J.M. Kernbach, Letter to the Editor. Importance of calibration assessment in machine learning-based predictive analytics. J. Neurosurg. Spine 32, 985-987 (2020). https://doi.org/ 10.3171/2019.12.SPINE191503

38. F.G. Barker, A. Klibanski, B. Swearingen, Transsphenoidal surgery for pituitary tumors in the United States, 1996-2000: mortality, morbidity, and the effects of hospital and surgeon volume. J. Clin. Endocrinol. Metab. 88, 4709-4719 (2003). https://doi.org/ 10.1210/jc. 2003-030461

39. T. Hastie, R. Tibshirani, J. Friedman, The Elements of Statistical Learning: Data Mining, Inference, and Prediction, 2nd edn. (Springer Science \& Business Media, 2009)

40. J.M. Kernbach, V.E. Staartjes, Machine learning-based clinical prediction modeling - a practical guide for clinicians. ArXiv200615069 Cs Stat. (2020)

41. M.A. Mooney, D.A. Hardesty, J.P. Sheehy, C.R. Bird, K. Chapple, W.L. White, A.S. Little, Rater reliability of the Hardy classification for pituitary adenomas in the magnetic resonance imaging era. J. Neurol. Surg. B Skull Base 78, 413-418 (2017). https://doi.org/10.1055/s-0037-1603649

42. M.A. Mooney, D.A. Hardesty, J.P. Sheehy, R. Bird, K. Chapple, W.L. White, A.S. Little, Interrater and intrarater reliability of the Knosp scale for pituitary adenoma grading. J. Neurosurg. 126, 1714-1719 (2017). https://doi.org/10.3171/2016.3.JNS153044 